\title{
A new interpretation of the integer and real WZ factorization using block scaled ABS algorithms
}

\author{
Effat Golpar-Raboky ${ }^{1}$, Nezam Mahdavi-Amiri ${ }^{2,}$ * \\ ${ }^{1}$ Department of Mathematics, University of Qom, Qom, Iran \\ ${ }^{2}$ Faculty of Mathematical Sciences, Sharif University of Technology, Tehran, Iran
}

Received 6 June 2013; Accepted 11 June 2014

Editor: Yunhai Xiao

\begin{abstract}
The $W Z$ factorization suitable for parallel computing was introduced by Evans. Here, a block generalization of the ABS class of methods for the solution of linear system of equations is given and it is shown that it covers the whole class of conjugate direction methods defined by Stewart. The methods produce a factorization of the coefficient matrix implicitly, generating well-known matrix factorizations. We show how to set the parameters of a block ABS algorithm to compute the $W Z$ and $Z W$ factorizations of a nonsingular matrix as well as the $W^{T} W$ and $Z^{T} Z$ factorizations of a symmetric positive definite matrix. We also provide a new interpretation of the necessary and sufficient conditions for the existence of the $W Z$ and $Z W$ factorizations of a nonsingular matrix. We show how to appropriate the parameters to construct algorithms for computing the $Q Z$ and the $Q W$ factorizations, where $Q^{T} Q$ is an $X$-matrix. We also provide the conditions for the existence of the integer $W Z$ and the integer $Z W$ factorizations of an integer matrix and compute the factorizations by the integer ABS algorithm.
\end{abstract}

Keywords ABS algorithm, $W Z$ factorization, $Z W$ factorization, $W^{T} W$ factorization, $Z^{T} Z$ factorization, $\mathrm{X}$ factorization, Cross orthogonal matrix.

DOI: $10.19139 /$ soic.v2i3.16

\footnotetext{
${ }^{*}$ Correspondence to: Tel: +98-021-66165607, E-mail addresses: nezamm@sharif.edu (Nezam Mahdavi-Amiri), g.raroky@qom.ac.ir (Effat Golpar-Raboky).
}

ISSN 2310-5070 (online) ISSN 2311-004X (print)

Copyright (c) 2014 International Academic Press 


\section{Introduction}

The central problem of linear algebra is the solution of linear system of equations $A x=b$. Direct methods used to solve a linear system of equations are based on a factorization of the coefficient matrix into factors that are easy to be used in solving equations. The Gaussian elimination process (i.e., the $L U$ factorization) is optimal among the methods involving only linear combinations of rows and columns [4].

Implicit matrix elimination schemes for solving linear systems were introduced by Evans [8] and Evans and Hatzopoulos [7]. These schemes propose the elimination of two matrix elements simultaneously (as opposed to a single element in Gaussian elimination) and is eminently suitable for parallel implimentation [9]. ABS class of algorithms was constructed for the solution of linear systems utilizing some basic ideas such as vector projection and rank one update techniques [1,3]. The ABS class was later extended to solve optimization problems [3] and systems of linear Diaphantine equations (see [5, 6, 17, 18]). Reviews of ABS methods and their extensions can be found in [21,22].

Consider the linear system

$$
A x=b, \quad x \in R^{n}, A \in R^{n \times n}, b \in R^{n} .
$$

For simplicity, assume that $A$ is a nonsingular matrix. A scaled version of the linear ABS class was introduced in [3]. Let $V \in R^{n \times n}$ be a nonsingular matrix. Obviously, the system (1) is equivalent to the following scaled system:

$$
V^{T} A x=V^{T} b .
$$

\section{Algorithm 1. Basic ABS algorithm.}

(1) Let $H_{1} \in R^{n \times n}$ be an arbitrary nonsingular matrix. Let $i=1$ and $r=0$.

(2) Compute $s_{i}=H_{i} a_{i}$.

(3) If $s_{i}=0$ (the $i$ th row of $A$ is dependent on its first $i$-1 rows) then let $H_{i+1}=H_{i}$ and go to (6).

(4) $\left(s_{i} \neq 0\right.$ and hence the $i$ th row of $A$ is independent of its first $i$-1 rows) Compute the search vectors $p_{i}$ by

$$
p_{i}=H_{i}^{T} f_{i},
$$

where $f_{i} \in R^{n}$ is arbitrary, save for the condition $s_{i}^{T} f_{i} \neq 0$. 
(5) (Updating the null space generator) Update $H_{i}$ by

$$
H_{i+1}=H_{i}-\frac{H_{i} a_{i} q_{i}^{T} H_{i}}{q_{i}^{T} H_{i} a_{i}},
$$

where $q_{i} \in R^{n}$ is an arbitrary vector satisfying $s_{i}^{T} q_{i} \neq 0$, and let $r=r+1$.

(6) If $i=m$ then Stop $\left(H_{m+1}^{T}\right.$ generates the null space of $A$ and $r$ is its rank) else let $i=i+1$ and go to (2).

The matrices $H_{i}$ are generalizations of projections matrices. The updating formula (4) for the $H_{i}$ is a rank reduction formula first appeared in a book by Wedderburn [23]. They have been named Abaffians since the First International Conference on ABS Methods (Luyoyang, China, 1991).

Considering (2), it is easily verified that the scaled ABS class is obtained by replacing $a_{i}$ with $A^{T} v_{i}$ [3] in Algorithm 1 . The algorithms produce a matrix factorization $V^{T} A P=L$, where $L$ is a lower triangular matrix (see [3]). Choices of the parameters $H_{1}, v_{i}, f_{i}$ and $q_{i}$ determine particular methods within the class so that various matrix factorizations such as LU, QR and Cholesky factorizations are derived [3]. Recently, Golpar-Raboky and Mahdavi-Amiri showed how to compute the Smith normal form of an integer matrix using the scaled integer ABS algorithm [14, 15]. Later Mahdavi-Amiri and Golpar-Raboky [19] extended the rank reducing process and developed a new class of algorithms containing the scaled extended ABS class of algorithms and the Wedderburn rank reducing process.

It has been proved in $[11,13]$ that all full rank factorizations can be produced only by a subset of the ABS methods.

The integer ABS (IABS) class of algorithms has been developed by Esmaeili et al. $[5,6]$ to compute the general integer solution of linear Diophantine equations. There, conditions for the existence of an integer solution and determination of all integer solutions of a linear Diophantine system have been established.

The block ABS algorithm, due to Abaffy and Galantai [2] for the scaled ABS class, further developed in several papers by Galantai, is a block form of the ABS algorithm [3]. Let $n_{1}, \cdots, n_{s}$ be positive integer numbers so that $n_{1}+\cdots+n_{s}=n$. Let $V$ be partitioned in the form $V=\left[V_{1}, \cdots, V_{s}\right]$, where $V_{i}, \in R^{n \times n_{i}}$. The block scaled ABS algorithm is as follows.

- Let $H_{1} \in R^{n \times n}$ be arbitrary and nonsingular. 
- For $i=1$ to $s$ do

- Compute $S_{i}=H_{i} A^{T} V_{i}$. Compute the matrix $P_{i}=H_{i}^{T} F_{i}$, where $F_{i} \in$ $R^{n \times n_{i}}$ is an arbitrary matrix so that $S_{i}^{T} F_{i}$ is nonsingular, and update $H_{i}$ by

$$
H_{i+1}=H_{i}-H_{i} A^{T} V_{i}\left(Q_{i}^{T} H_{i} A^{T} V_{i}\right)^{-1} Q_{i}^{T} H_{i},
$$

where $Q_{i} \in R^{n \times n_{i}}$ is an arbitrary matrix so that $S_{i}^{T} Q_{i}$ is nonsingular.

- Define $V_{i+1} \in R^{n \times n_{i+1}}$, an arbitrary full column rank matrix with column being linearly independent of columns of $V_{1}, \cdots, V_{i}$.

One main result of the ABS algorithms has been the derivation of the ABS class of algorithms for linear Diophantine equations given by Esmaeili, MahdaviAmiri and Spedicato [6] and its extension using the scaled ABS algorithms [21]. Each integer ABS algorithm decides whether the Diophantine system has an integer solution, and, if so, obtains a particular solution along with an integer matrix with possibly dependent rows generating the integer null space of the equations. Consider the linear Diophantine system:

$$
A x=b, \quad A \in Z^{m \times n}, x \in Z^{n}, b \in Z^{m}, m \leq n .
$$

The system (6) is equivalent to the scaled system,

$$
V^{T} A x=V^{T} b,
$$

where $V$ is an arbitrary $m$ by $m$ unimodular matrix, an integer matrix with its determinant being equal to +1 or -1 .

First, we recall some results from number theory and then present the steps of an integer ABS (IABS) algorithm.

Note that if $A$ is unimodular, then $A^{-1}$ is also unimodular.

\section{Definition 1}

A matrix $A$ is called totally unimodular if each square submatrix of $\mathrm{A}$ has determinant equal to $0,+1$, or -1 . In particular, each entry of a totally unimodular matrix is $0,+1$, or -1 .

\section{Theorem 1}

(Fundamental theorem of the single linear Diophantine equation) Let $a_{1}, \cdots, a_{n}$ and $b$ be integer numbers. The Diophantine linear equation $a_{1} x_{1}+\cdots+a_{n} x_{n}=b$ has an integer solution if and only if $\operatorname{gcd}\left(a_{1}, \cdots, a_{n}\right) \mid b$ (if $n>1$, then there are an infinite number of integer solutions). 
The scaled integer ABS algorithm (SIABS) has the following structure, with $\operatorname{gcd}(u)$ being the greatest common divisor of a vector $u$.

- Let $H_{1} \in Z^{n \times n}$ be arbitrary and unimodular.

- For $i=1$ to $n$ do

- Compute $s_{i}=H_{i} A^{T} v_{i}$.

- Compute $\delta_{i}=\operatorname{gcd}\left(s_{i}\right)$ and $p_{i}=H_{i}^{T} f_{i}$, where, $f_{i} \in Z^{n}$ is an arbitrary integer vector satisfying $s_{i}^{T} f_{i}=\delta_{i}$. Update $H_{i}$ by

$$
H_{i+1}=H_{i}-\frac{H_{i} A^{T} v_{i} q_{i}^{T} H_{i}}{q_{i}^{T} H_{i} A^{T} v_{i}},
$$

where $q_{i} \in Z^{n}$ is an arbitrary integer vector satisfying $s_{i}^{T} q_{i}=\delta_{i}$.

- Define $v_{i+1} \in Z^{n}$ so that $v_{1}, \cdots, v_{i+1}$ make the columns of a unimodular matrix.

The $W Z$ factorization corresponds to a version of the block $L U$ algorithm, with block sizes equal to two. The $W$-matrix and $Z$-matrix are constructed by applying appropriate permutation matrices on rows and columns of a lower triangular matrix.

Here, making use of permutations in the block ABS method we compute the $W Z$ and $W Z$ factorizations of a nonsingulaer matrix as well as the $W^{T} W$ and the $Z^{T} Z$ factorizations of a symmetric positive definite matrix. We also show how to compute the $Q Z$ and the $Q W$ factorizations, where $Q^{T} Q$ is an $X$-matrix, using the ABS algorithms.

The remainder of our work is organized as follows. In Section 3, we explaine the $W Z$ factorization. In Section 2, we present a new formulation of the neseccary and sufficient conditions for existence of the real and integer $W Z$ factorizations. Then, we present an algorithm for computing the $W Z$ factorization as well as the $W^{T} W$ factorization of a positive definite matrix using the block scaled ABS algorithm. An algorithm for computing the $Q Z$ factorization where $Q^{T} Q$ is an $X$-matrix is also given. In Section 3, we compute the real and integer $Z W$ factorizations by appropriate setting of the parameters of the block scaled ABS algorithm. We also compute the $Z^{T} Z$ factorization of a positive definite matrix and the $Q W$ factorization, where $Q^{T} Q$ is an $X$-matrix, using the block scaled ABS algorithms. Necessary and sufficient conditions for existence of $Z W$ factorization based on the block ABS algorithm are also given. We conclude in Section 4. 


\section{WZ factorization using the block scaled ABS algorithm}

To solve a system of linear equations, the $W Z$ factorization splitting procedure proposed in $[8,9]$ is convenient for parallel computing. The $W Z$ factorization offers a parallel method for solving dense linear systems, where $A$ is a square $n \times n$ matrix, and $b$ is an $n$-vector.

\section{Definition 2}

Let $\mathrm{s}$ be a real number, and denote by $\lfloor s\rfloor(\lceil s\rceil)$, the greatest (least) integer less (bigger) than or equal to $s$.

\section{Definition 3}

We say that a matrix $A$ is factorized in the form $W Z$ if

$$
A=W Z,
$$

where the matrices $W$ and $Z$ have the following structures:

$$
W=\left(\begin{array}{lllll}
1 & 0 & 0 & 0 & \bullet \\
\bullet & 1 & 0 & \bullet & \bullet \\
\bullet & \bullet & 1 & \bullet & \bullet \\
\bullet & \bullet & 0 & 1 & \bullet \\
\bullet & 0 & 0 & 0 & 1
\end{array}\right), Z=\left(\begin{array}{ccccc}
\bullet & \bullet & \bullet & \bullet & \bullet \\
0 & \bullet & \bullet & \bullet & 0 \\
0 & 0 & \bullet & 0 & 0 \\
0 & \bullet & \bullet & \bullet & 0 \\
\bullet & \bullet & \bullet & \bullet & \bullet
\end{array}\right)
$$

with the empty bullets standing for zero and the other bullets standing for possible nonzeros.

\section{Definition 4}

We define an X-matrix as follows:

$$
X=\left(\begin{array}{lllll}
\bullet & 0 & 0 & 0 & \bullet \\
0 & \bullet & 0 & \bullet & 0 \\
0 & 0 & \bullet & 0 & 0 \\
0 & \bullet & 0 & \bullet & 0 \\
\bullet & 0 & 0 & 0 & \bullet
\end{array}\right)
$$

The following theorems express the conditions for the existence of a WZ factorization of a nonsigular matrix (see [20]). Later, we give a new set of conditions useful for our purposes.

The well-known $L U$ factorization is one of the most commonly used algorithms to solve linear systems and the $W Z$ factorization is an interesting variation of the block $L U$ factorization with block sizes equal to two. Assume that $P=\left[p_{\delta_{1}}, \cdots, p_{\delta_{n}}\right]$ is a permutation matrix so that $p_{\delta_{i}}=i$, if $i$ is odd, and $p_{\delta_{i}}=n-i+1$, if $i$ is even, $i=1, \cdots, n / 2$, with $n$ being even. Let $L$ and $U$ be the block lower and upper triangular matrices with block sizes equal to two, 
respectively. Then, $P^{-T} L P^{T}$ is a $W$-matrix and $P^{-T} U P^{T}$ is a $Z$-matrix.

We know that the matrix $A$ has an $L U$ decomposition if and only if the leading principal block submatrices of $A$ are nonsingular; see [12]. Therefore, we have the following result.

Theorem 2

(Factorization Theorem). Let $A \in R^{n \times n}$ be nonsingular. The matrix $A$ has a $W Z$ factorization if and only if for every $k, 1 \leq k \leq s$, with $s=\lfloor n / 2\rfloor$, if $n$ is even, and $s=\lceil n / 2\rceil$, if $n$ is odd, the submatrix

$$
\Delta_{k}=\left(\begin{array}{cccccc}
a_{1,1} & \cdots & a_{1, k} & a_{1, n-k+1} & \cdots & a_{1, n} \\
\vdots & \cdots & \vdots & \vdots & \cdots & \vdots \\
a_{k, 1} & \cdots & a_{k, k} & a_{k, n-k+1} & \cdots & a_{k, n} \\
a_{n-k+1,1} & \cdots & a_{n-k+1, k} & a_{n-k+1, n-k+1} & \cdots & a_{n-k+1, n} \\
\vdots & \ldots & \vdots & \vdots & \ldots & \vdots \\
a_{n, 1} & \cdots & a_{n, k} & a_{n, n-k+1} & \cdots & a_{n, n}
\end{array}\right)_{2 k, 2 k}
$$

of $A$ is invertible. Moreover, the factorization is unique.

\section{Proof}

See Theorem 2 in [20].

The following results have also been established in [20].

- If $A \in R^{n \times n}$ is nonsingular, then a $W Z$ factorization can always be obtained by pivoting. That is, there exists a row permutation matrix $\Pi$ and the factors $W$ and $Z$ such that

$$
\Pi A=W Z .
$$

- Every symmetric positive definite matrix has a $W Z$ factorization.

When $A$ is a symmetric positive definite matrix, it is possible to factor $A$ in the Cholesky factorization form $A=L L^{T}$, for some lower triangular matrix $L$. A variant of the classical Cholesky factorization, called Cholesky QIF, is given by Evans [10]. Existence and stability of this factorization are proved by Khazal [16].

Here, we propose a new formulation of Theorem 2 using the block scaled ABS algorithm.

\section{Theorem 3}

Let $A \in Z^{n \times n}$ be nonsingular. If the $\Delta_{k}, k=1, \cdots, n / 2$, are unimodular, then 
the block ABS algorithm with parameter choices $H_{1}=I, Q_{i}=V_{i}=\left[e_{i}, e_{n-i+1}\right]$ is well defined and the implicit factorization $V^{T} A P$ with $p_{i}=H_{i}^{T} e_{i}, p_{n-i+1}=$ $H_{i}^{T} e_{n-i+1}, i=1, \cdots, n / 2$, and $V=\left[V_{1}, \cdots, V_{n / 2}\right]$ leads to a $W Z$ factorization.

\section{Proof}

Let $H_{1}=I, V_{i}=\left[v_{2 i-1}, v_{2 i}\right]=\left[e_{i}, e_{n-i+1}\right], Q_{i}=\left[q_{2 i-1}, q_{2 i}\right]=\left[e_{i}, e_{n-i+1}\right]$ and $H_{i+1}$ be defined by (5). Then, according to Theorem 5.5 in [3], we have

$$
H_{i+1}=\left[\begin{array}{ccc}
0 & 0 & 0 \\
K_{i} & I_{n-2 i} & L_{i} \\
0 & 0 & 0
\end{array}\right]
$$

with $\quad K_{i}, L_{i} \in Z^{n-2 i, i} . \quad$ Let $\quad P_{i}=\left[p_{2 i-1}, p_{2 i}\right]=H_{i}^{T}\left[e_{i}, e_{n-i+1}\right], \quad P=$ $\left[P_{1}, \cdots, P_{n / 2}\right]$ and $V=\left[V_{1}, \cdots, V_{n / 2}\right]$. Then, the block ABS algorithm produces $V^{T} A P=L$, where $P$ is an upper triangular matrix and $L$ is a lower triangular matrix. Now, we have

$$
V^{T} A P=L \Rightarrow A P V^{T}=V^{-T} L V^{T} \Rightarrow A Z=W
$$

where $Z=P V^{T}$ is an $Z$-matrix, with 1 as diagonal and 0 as off diagonal entries, and $W=V^{-T} L V^{T}$ is an $W$-matrix.

Next, we present an algorithm for computing the $W Z$ factorization.

\section{Algorithm 2. The WZ factorization.}

(1) Let $H_{1}=I_{n}$ and $i=1$.

(2) Let $A_{i}=\left[a_{i}, a_{n-i+1}\right]^{T}$ and $\left[p_{i}, p_{n-i+1}\right]=H_{i}^{T}\left[e_{i}, e_{n-i+1}\right]$.

(4) Let $Q_{i}=\left[e_{i}, e_{n-i+1}\right]$. Update $H_{i}$ by

$$
H_{i+1}=H_{i}-H_{i} A_{i}\left(Q_{i}^{T} H_{i} A_{i}\right)^{-1} Q_{i}^{T} H_{i} .
$$

(5) Let $i=i+1$. If $i \leq n / 2$ then go to (2).

(6) Compute $A P=W$, where $P=\left[p_{1}, \cdots, p_{n}\right]$. Stop.

We now have the following results.

\section{Theorem 4}

Let $A$ be a symmetric positive definite matrix. Then, there exists a $Z^{T} D Z$ factorization for $A$, obtained by the ABS algorithm. 
Proof

Consider the assumptions of Theorem 3 and let $V_{i}=P_{i}$, for $i=1, \cdots s$. Then, $V^{T} A P$ is an $X$-matrix, $V$ and $P$ are $Z$-matrices and we have

$$
V^{T} A P=X \Rightarrow A=V^{-T} X P^{-1}=Z^{T} D Z,
$$

where $D$ is an $X$-matrix, $Z^{T}=V^{-T}$ and $Z=P^{-1}$.

\section{Theorem 5}

Let $A$ be symmetric positive definite. Then, there exists a $Z^{T} Z$ factorization for $A$, obtained by the ABS algorithm.

Proof

Consider the assumptions of Theorem 3 and let $V_{i}=P_{i}$, for $i=1, \cdots s$. Then, $V^{T} A P$ is an $\mathrm{X}$-matrix, $V$ and $P$ are $Z$-matrices and we have

$$
V^{T} A P=X \Rightarrow A=V^{-T} X P^{-1}=Z^{T} Z,
$$

where $X$ is an $X$-matrix, $Z^{T}=V^{-T} X^{1 / 2}$ and $Z=X^{1 / 2} P^{-1}$.

\section{Remark 1}

Now, let $A \in Z^{n \times n}, H_{1}=I$ and $\Delta_{k}, k=1, \cdots, n / 2$, are unimodular. Then, the sequence of $H_{i+1}, i=1, \cdots, s$ generated by the Algorithm 2 are integer matrices [5] and the Algorithm 2, leads to a $W Z$ factorization, where the $W$ and the $Z$ are integer $W$-matrix and $Z$-matrix, respectively.

\subsection{The QZ Algorithm}

\section{Definition 5}

A matrix $Q$ is said a cross orthogonal matrix if $Q^{T} Q$ is an $X$-matrix.

\section{Definition 6}

We say that a matrix $A$ is factorized in the form $Q Z$ if

$$
A=Q Z,
$$

where the matrix $Z$ is a $Z$-matrix and $Q$ is a cross orthogonal matrix.

\section{Theorem 6}

Let $A \in R^{n \times n}, \quad H_{1}=I, \quad V_{i}=\left[e_{i}, e_{n-i+1}\right], \quad Q_{i}=F_{i}=A^{T} V_{i} \quad$ and $\quad P V_{i}=$ $H_{i}^{T} A^{T} V_{i}, i=1, \cdots, s$, with $s=\lfloor n / 2\rfloor$, if $n$ is even, and $s=\lceil n / 2\rceil$, if $n$ is odd. Then, there exists a $Q Z$ factorization, obtained by the block scaled ABS algorithm.

Proof

Making use of the proof of Theorem 5.1 in [3], $P$ is a cross orthogonal matrix and we have 


$$
A P=W \Rightarrow A^{T}=Q Z
$$

where $Z=W^{T}$ is a $Z$-matrix , $Q=P^{-T}$ and $Q$ is a cross orthogonal matrix. Thus, we admit a $Q Z$ factorization for $A^{T}$. Of course, a $Q Z$ factorization for $A$ is easily found by applying the above process to $A^{T}$.

\section{3. $Z W$ factorization using the block scaled ABS algorithm}

Here, we present conditions for the existence and compute the $Z W$ factorization of a nonsingular matrix using the ABS algorithm.

\section{Definition 7}

We say that a matrix $A$ is factorized in the form $Z W$ if

$$
A=Z W
$$

where the matrices $W$ and $Z$ have the following structures:

$$
Z=\left(\begin{array}{lllll}
1 & \bullet & \bullet & \bullet & \bullet \\
0 & 1 & \bullet & \bullet & 0 \\
0 & 0 & 1 & 0 & 0 \\
0 & \bullet & \bullet & 1 & 0 \\
\bullet & \bullet & \bullet & \bullet & 1
\end{array}\right), W=\left(\begin{array}{ccccc}
\bullet & 0 & 0 & 0 & \bullet \\
\bullet & \bullet & 0 & \bullet & \bullet \\
\bullet & \bullet & \bullet & \bullet & \bullet \\
\bullet & \bullet & 0 & \bullet & \bullet \\
\bullet & 0 & 0 & 0 & \bullet
\end{array}\right)
$$

Now, we give a new characterization of the necessary and sufficient conditions for the $Z W$ factorization.

\section{Theorem 7}

Let $A \in R^{n, n}$ be a nonsingular matrix. $A$ has a $Z W$ factorization if and only if for every $k, 1 \leq k \leq s$, with $s=\lfloor n / 2\rfloor$, if $n$ is even, and $s=\lceil n / 2\rceil$, if $n$ is odd, the submatrix

$$
\Lambda_{k}=\left(\begin{array}{cccc}
a_{s-k+1, s-k+1} & \cdots & & a_{s-k+1, s+k} \\
\vdots & \cdots & \vdots & \\
a_{s+k, s-k+1} & \cdots & & a_{s+k, s+k}
\end{array}\right)_{2 k, 2 k}
$$

is invertible. Moreover, the factorization is unique.

Proof

The proof follows the lines of the proof for Theorem 5.5 in [3] replacing $\Delta_{i}$ by $\Lambda_{i}$.

Next, we propose a new formulation of Theorem 7 using the block scaled ABS algorithm. 


\section{Theorem 8}

Let $A \in Z^{n \times n}$ be nonsingular. If $\Delta_{k}, k=1, \cdots, n / 2$, is nonsingular, then the block ABS algorithm with parameter choices $H_{1}=I, Q_{i}=V_{i}=\left[e_{\frac{n}{2}-i+1}, e_{\frac{n}{2}+i}\right]$ is well defined and the implicit factorization $V^{T} A P$ with $p_{\frac{n}{2}-i+1}=$ $H_{i}^{T} e_{\frac{n}{2}-i+1}, p_{\frac{n}{2}+i}=H_{i}^{T} e_{\frac{n}{2}+i}, i=1, \cdots, n / 2$, and $V=\left[V_{1}, \cdots, V_{n / 2}\right]$ leads to a $Z W$ factorization.

\section{Proof}

Let $\quad H_{1}=I, \quad V_{i}=\left[v_{2 i-1}, v_{2 i}\right]=\left[e_{\frac{n}{2}-i+1}, e_{\frac{n}{2}+i}\right], \quad Q_{i}=\left[q_{2 i-1}, q_{2 i}\right]=$ $\left[e_{\frac{n}{2}-i+1}, e_{\frac{n}{2}+i}\right]$ and $H_{i+1}$ be defined by (5). Then, according to Theorem 5.5 in [3], we have

$$
H_{i+1}=\left[\begin{array}{ccc}
I_{i} & K_{2 i} & 0 \\
0 & 0 & 0 \\
0 & L_{2 i} & I_{i}
\end{array}\right],
$$

with $\quad K_{i}, L_{i} \in R^{n-2 i, 2 i}$. Let $\quad P_{i}=\left[p_{2 i-1}, p_{2 i}\right]=H_{i}^{T}\left[e_{\frac{n}{2}-i+1}, e_{\frac{n}{2}+i}\right], \quad P=$ $\left[P_{1}, \cdots, P_{n / 2}\right]$ and $V=\left[V_{1}, \cdots, V_{n / 2}\right]$. Then, the block ABS algorithm produces $V^{T} A P=L$, where $P$ is upper triangular and $L$ is lower triangular. Now, we have

$$
V^{T} A P=L \Rightarrow A P V^{T}=V^{-T} L V^{T} \Rightarrow A Z=W,
$$

where $W=P V^{T}$ is a $W$-matrix with 1 as diagonal and 0 as off diagonal entries, and $W=V^{-T} L V^{T}$ is a $Z$-matrix.

Now, we are ready to present an algorithm for computing the $W Z$ factorization.

\section{Algorithm 3. The $Z W$ factorization.}

(1) Let $H_{1}=I_{n}$ and $i=1$.

(2) Let $A_{i}=\left[a_{\frac{n}{2}-i+1}, a_{\frac{n}{2}+i}\right]^{T}$ and $\left[p_{\frac{n}{2}-i+1}, p_{\frac{n}{2}+i}\right]=H_{i}^{T}\left[e_{\frac{n}{2}-i+1}, e_{\frac{n}{2}+i}\right]$.

(4) Let $Q_{i}=\left[e_{\frac{n}{2}-i+1}, e_{\frac{n}{2}+i}\right]$. Update $H_{i}$ by

$$
H_{i+1}=H_{i}-H_{i} A_{i}\left(Q_{i}^{T} H_{i} A_{i}\right)^{-1} Q_{i}^{T} H_{i},
$$

(5) Let $i=i+1$. If $i \leq n / 2$ go to (2).

(6) Compute $A P=Z$, where $P=\left[p_{1}, \cdots, p_{n}\right]$. Stop.

\section{Theorem 9}

Let $A$ be symmetric positive definite. Then, there exists a $W^{T} W$ factorization for $A$, obtained by the ABS algorithm. 
Proof

Consider the assumption of Theorem 8 and let $V_{i}=P_{i}$, for $i=1, \cdots s$. Then, $V^{T} A P$ is an $X$-matrix, $V$ and $P$ are $W$-matrices and we have

$$
V^{T} A P=X \Rightarrow A=V^{-T} X P^{-1}=W^{T} W
$$

where $X$ is an $X$-matrix, $W^{T}=V^{-T} X^{1 / 2}$ and $W=X^{1 / 2} P^{-1}$.

\section{Remark 2}

Now, let $A \in Z^{n \times n}, H_{1}=I$ and $\Delta_{k}, k=1, \cdots, n / 2$, are unimodular. Then, the sequence of $H_{i+1}, i=1, \cdots, s$ generated by the Algorithm 3 are integer matrices [5] and the algorithm leads to a $Z W$ factorization, where the $W$ and the $Z$ are integer $W$-matrix and $Z$-matrix, respectively.

\subsection{The $Q W$ Algorithm}

\section{Definition 8}

We say that a matrix $A$ is factorized in the form $Q W$ if

$$
A=Q W
$$

where the matrix $W$ is a $W$-matrix and $Q$ is a cross orthogonal matrix.

\section{Theorem 10}

Let $A \in R^{n \times n}, \quad H_{1}=I, \quad V_{i}=\left[e_{s-i+1}, e_{s+i}\right], \quad Q_{i}=F_{i}=A^{T} V_{i}$ and $P V_{i}=$ $H_{i}^{T} A^{T} V_{i}, i=1, \cdots, s$, with $s=\lfloor n / 2\rfloor$, if $n$ is even, and $s=\lceil n / 2\rceil$, if $n$ is odd,. Then, there exists a $Q Z$ factorization, obtained by the block scaled ABS algorithm.

\section{Proof}

Making use of the proof of Theorem 5.1 in [3], $P$ is a cross orthogonal matrix and we have

$$
A P=Z \Rightarrow A^{T}=Q W
$$

where $W=Z^{T}$ is a $W$-matrix and $Q=P^{-T}$ is a cross orthogonal matrix. Thus, we admit a $Q W$ factorization for $A^{T}$. Of course, a $Q W$ factorization for $A$ is easily found by applying the above process to $A^{T}$.

\section{Conclusions}

We showed how to appropriate the parameters of the block scaled ABS algorithms to construct algorithms for computing the $W Z$ and $Z W$ factorizations of a nonsingular matrix and the $W^{T} W$ and $Z^{T} Z$ factorizations of a symmetric positives definite matrix. New formulation of the necessary and sufficient 
conditions for the existence of the $W Z$ and the $Z W$ factorizations of a nonsingular matrix were given. We also derived two new factorizations, the $Q Z$ and $Q W$, with $Q^{T} Q$ being an $X$-matrix, and showed how to compute the factorizations using the block scaled ABS algorithms. We also provided the conditions for the existence of the integer $W Z$ and $Z W$ factorizations of an integer matrix and computated the factorizations by the integer ABS algorithms.

\section{Acknowledgements}

The second author thanks the Research Council of Sharif University of Technology for supporting this work.

\section{REFERENCES}

1. J. Abaffy, C.G. Broyden and E. Spedicato, A class of direct methods for linear equations, Numer. Math. 45 (1984) 361-378.

2. J. Abaffy and A. Galantai, Conjugate direction methods for linear and nonlinear systems of algebraic equations, Colloq. Math. Soc. Janos Bolyai, Numerical Methods, Miskolc 50 (1986) 481-502.

3. J. Abafy and E. Spedicato, ABS Projection Algorithms, Mathematical Techniques for Linear and Nonlinear Equations, Ellis Horwood, Chichester, 1989.

4. G.W. Althaus and D.J. Evans, Algorithms for Large Scale Linear Algebraic Systems Applications in Science and Engineering, Kluwer Academic Publishers, pp. 37-53.

5. H. Esmaeili, N. Mahdavi-Amiri and E. Spedicato, Generationg the integer null space and conditions for determination of an integer basis using the ABS algorithms, Bulletin of the Iranian Mathematical Society 27 (2001) 1-18.

6. H. Esmaeili, N. Mahdavi-Amiri and E. Spedicato, A class of ABS algorithms for Diophantine linear systems, Numer. Math. 90 (2001) 101-115.

7. D.J. Evans and M. Hatzopoulos, A parallel linear system solver, International Journal of Computer Mathematics 7 (1979) 227-238.

8. D.J. Evans, Implicit matrix elimination schemes, International Journal of Computer Mathematics 48 (1993) 229-237.

9. D.J. Evans and R. Abdullah, The paralle implicit elimination (PIE) method for the solution of linear systems, Parallel Algorithms and Applications 4 (1994) 153-162.

10. D.J. Evans, The Cholesky QIF algorithm for solving symmetric linear systems, International Journal of Computer Mathematics 72 (1999) 283-285.

11. A. Galantai, Rank reduction, factorization and conjugation, Linear and Multilinear Algebra 49 (2001) 195-207.

12. A. Galantai, Projection methods for linear and nonlinear equations, Dissertation submitted to the Hungarian Academy of Sciences for the degree "MTA Doktora", University of Miskolc, 2003.

13. A. Galantai, Projectors and Projection Methods, Kluwer, 2004.

14. E. Golpar-Raboky and N. Mahdavi-Amiri, Diophantine quadratic equation and Smith normal form using scaled extended integer integer ABS algorithms, Journal of Optimization Theory and Applications 152(1) (2012) 75-96.

15. E. Golpar-Raboky and N. Mahdavi-Amiri, Extended integer rank reduction formulas containing Wedderburn and Abaffy-Broyden-Spedicato rank reducing processes, Linear and Multilinear Algebra 61(12) (2013) 1641-1659. 
16. R.R. Khazal, Existence and stability of Cholesky QIF for symmetric linear systems, International Journal of Computer Mathematics 79 (2002) 1013-1023.

17. M. Khorramizadeh and N. Mahdavi-Amiri, Integer extended ABS algorithms and possible control of intermediate results for linear Diophantine systems, 4OR 7 (2009) 145-167.

18. M. Khorramizadeh and N. Mahdavi-Amiri, On solving linear Diophantine systems using generalized Rosser's algorithm, Bulletin of the Iranian Mathematical Society 34(2) (2008) $1-25$.

19. N. Mahdavi-Amiri and E. Golpar-Raboky, Extended rank reduction formulas containing Wedderburn and Abaffy-Broyden-Spedicato rank reducing processes, Linear Algebra and its Applications 439(11) (2013) 3318-3331.

20. S.C.S. Rao, Existence and uniqueness of WZ factorization, Parallel Computing 23 (1997) 1129-1139.

21. E. Spedicato, E. Bodon, A. Del Popolo and N. Mahdavi-Amiri, ABS methods and ABSPACK for linear systems and optimization: A review, 4OR 1 (2003) 51-66.

22. E. Spedicato, E. Bodon, Z. Xia and N. Mahdavi-Amiri, ABS methods for continuous and integer linear equations and optimization, CEJOR 18 (2010) 73-95.

23. J.H.M. Wedderburn, Lectures on Matrices, Colloquium Publications, American Mathematical Society, New York, 1934. 